\section{Patterns of antibiotic prescriptions and appropriateness in the emergency room in a major secondary care hospital in Bahrain}

Jameela Mohammed al Salman, Sughra Alawi, Ebtihal Alyusuf, Eman Albasri, Shurooq Almarzooq, Fatma Alnashaba, Ali alahmed, Zahra Ali, Rawdha fardan, Ebtihal alyusuf

Department of internal medicine, Salmanyia Medical Complex Ministry of Health, PO box 12, Manama, Kingdome of Bahrain

Objective: To describe the pattern and appropriateness of antibiotics prescribed in the emergency room in a major secondary care hospital in Bahrain.

Methods: Patients aged more than or equal to 14 years old that attended emergency room and was prescribed antibiotics from 1 to 31 July 2014 were included. Data were obtained from patients' emergency records. Antibiotic treatment was classified to appropriate, inappropriate or unjustified use according to the local or international guidelines. Chi Square was performed to evaluate the variables associated to appropriateness antibiotic treatment.

Results: A total of 1313 patients were included (52.6\% males), mostly in the age group $14-30$ years old (45.3\%). The most frequent diseases attended were upper respiratory and urinary tract infections (27.3\% and $22.1 \%$,respectively). Cefuroxime was the most prescribed antibiotic (37.5\%) followed by ciprofloxacin (20.8\%). Percentage of inappropriate antibiotics prescription was $81.9 \%$ mostly due to unjustified use. Inappropriate antibiotic treatment was significantly more common in males (87.1\%; P-value $<0.001$ ), in patients without mentioned diagnosis, then upper respiratory tract infection (100\%, 96.9\%. P-value <0.001) and prescriptions written by emergency physicians (85.5\%; P-value <0.001).

Conclusion: The study concludes that high rate of inappropriate antibiotics use mostly among patients treated by emergency doctors.
Corresponding author:

\section{Dr. Jameela Alsalman}

”; jsalman@health.gov.bh 
Key words: Antibiotics appropriateness, pattern, Antibiotic guidelines, emergency department.

\section{Introduction}

Antibiotic resistance is a mounting public health concern [1], therefore it is important to preserve the effectiveness of presenting available antibiotic for future generations [2]. Antibiotic resistance was most commonly found in prescribed drug in several national and international studies [3].

The united states' chief medical officers annual reports of 2011, promoted the concepts of antimicrobial stewardship, which means that unnecessary or inappropriate use of antibiotics, should be avoided to minimize the selection of antibiotic resistant organisms [4]. In addition, the overuse of antibiotic drugs can lead to unnecessary side effects $[5,6,7]$. An estimated 142,500 visits were recorded to United States emergency departments each year because of adverse events associated with use of systemic antibiotics; approximately $80 \%$ due to allergic reactions $[5,6]$.

Studies on antibiotic appropriateness in emergency department settings are very limited and largely focused on in-patients and pediatrics age groups $[8,9,10]$.

There are many challenges associated with providing a systematic review for proper antibiotic prescription in emergency departments. These include rapid patients turn over, high rate of examined patients in emergency department [11], failure to reach final diagnosis and the physicians desire to maintain patient's satisfaction.

The aim of this study was to evaluate the patterns and appropriateness of antibiotics prescription, and to apply the stewardship program in emergency room in Salmaniya Medical Complex.

\section{Methods and population}

This is a prospective observational study included data collected on a daily basis from patients attending the accident and emergency room over a one-month period, from 1 to 31 July 2015.

The study was conducted in Salmaniya Medical Complex emergency room, which is the main secondary hospital in the Kingdom of Bahrain. The emergency room in the hospital examine an average 1000 patients per day. All Patients aged $\geq 14$ years old and who were prescribed antibiotics in the emergency room were included in the study. A total of 1313 patient's emergency room records were reviewed by the researchers. This study was approved by the research committee of the Ministry of Health in Bahrain

\section{Data collection}

A form was designed by the research team and were filled accordingly. The form included the following demographic data: sex, age, pregnancy status and nationality, vital signs, physical signs and symptoms, presenting system, laboratory and radiological investigations, antibiotic prescribed with their doses and duration, frequency and route, physician specialty and final diagnosis by the attending physician. No attempt was made to verify the accuracy of the physician diagnosis because the aim was to assess the physicians attitudes in prescribing antibiotic appropriateness in the emergency department. The antibiotic therapy was reviewed by each researcher to assure compliance with the recommendation of the local guidelines of infectious disease department at Ministry of Health in Bahrain [12] or the IDSA guidelines [13]. 
The following classification was used: Appropriate or inappropriate. The appropriate use was defined as proper antibiotic choice, dose, frequency, duration, route and combination. The inappropriate use was defined as improper antibiotic choice, dose, frequency, duration or route, improper combination or unjustified use. Patients who received antibiotics without mentioned diagnosis were considered inappropriate and unjustified use of antibiotics.

\section{Statistical analysis}

The statistical package for social science (SPSS) program version 21 was used for data entry and analysis. Frequencies and percentages were used to present qualitative variables. Also cross-tabulation was used to present the relationship between two qualitative variables. Chi- squared test was used to test whether there is a significant relationship between two categorical variables. $\mathrm{P}$ value $<0.05$ was considered statistically significant.

\section{Results}

The demographic characteristics of the patients are shown in Table 1. There were 690 males (52.6\%), of these 595 (45.3\%) belonged to age group 14-30year, there was no statistical significance difference among the different age groups or the nationality and the vast majority were Bahraini ( $n=1224$; $93.2 \%$ ) as shown in Table 1. Culture specimens were requested from part of the patients as followed: Urine cultures ( $\mathrm{n}=113 ; 8.6 \%$ ), blood cultures ( $n=32 ; 2.4 \%)$, wound swabs $(n=31 ; 2.36 \%)$ and vaginal swabs ( $n=26 ; 1.98 \%)$. The results of cultures showed bacterial growth in $20 \%, 4 \%, 10 \%, 12 \%$, respectively. Most patients were diagnosed with diseases of upper respiratory tract system $(n=358$; $27.3 \%)$, followed by renal system ( $n=290 ; 22.1 \%$ ) and skin and soft tissue ( $n=249 ; 19.0 \%)$, genital tract $(n=51,4 \%)$, lower respiratory tract infection ( $n=30,2 \%)$, musculoskeletal system $(n=26,2 \%)$
Table1. Demographical data $(n=1313)$

\begin{tabular}{|c|c|c|c|c|}
\hline \multicolumn{2}{|c|}{ Characteristics } & N & $\%$ \\
\hline \multirow{4}{*}{ Gender } & Male & 690 & 52.6 \\
& Female & 623 & 47.4 \\
& Total & $\mathbf{1 3 1 3}$ & $\mathbf{1 0 0}$ \\
\hline \multirow{4}{*}{ Pregnancy } & Yes & 151 & 24.2 \\
& No & 472 & 75.8 \\
& Total & $\mathbf{6 2 3}$ & $\mathbf{1 0 0}$ \\
& $14-30$ & 595 & 45.3 \\
\hline \multirow{4}{*}{ Age group in } & $31-40$ & 340 & 25.9 \\
years & $41-50$ & 154 & 11.7 \\
& $51-60$ & 109 & 8.3 \\
& $>60$ & 115 & 8.8 \\
& Total & $\mathbf{1 3 1 3}$ & $\mathbf{1 0 0}$ \\
& Bahraini & 1224 & 93.2 \\
\hline \multirow{5}{*}{ Nationality } & Non & 89 & 6.8 \\
& Bahraini & & \\
& Total & $\mathbf{1 3 1 3}$ & $\mathbf{1 0 0}$ \\
\hline
\end{tabular}

and others ( $n=27,2 \%)$, while $118(100.0 \%)$ were prescribed antimicrobials without clinical diagnosis as shown in Table 2. Emergency physicians wrote most of the prescriptions ( $n=1031$; 78.5\%), compared with other specialties including: obstetrics and gynecologist ( $n=196 ; 14.9 \%$ ), surgeons ( $n=43 ; 3.3 \%)$, internist $(n=15 ; 1.1 \%)$ and other specialties ( $n=28 ; 2.1 \%$ ) as demonstrated in Table 2. Chi squared $p$ value showed a high significant difference in appropriateness percentage between different presenting systems and prescribed specialties. Inappropriateness was more in those without mentioned diagnosis ( $\mathrm{n}=118,100 \%$ ) followed by upper respiratory tract system ( $n=347$; 96.9\%) as shown in Table 2. Emergency physicians dominate the most inappropriate prescriptions ( $\mathrm{n}=881 ; 85.5 \%$, P-value <0.001 ) (Table 2).

Out of 8358 patients visited the emergency department in July 2014, 1313 (7.2\%) were prescribed antibiotics. The most frequently prescribed antibiotics were cefuroxime ( $n=492 ; 37.5 \%$ ), ciprofloxacin ( $n=273 ; 20.8 \%$ ) and amoxicillin- 
Table 2. Appropriateness in relation to the involved body sites and the prescribing physician's specialty

\begin{tabular}{|c|c|c|c|c|c|}
\hline \multirow{3}{*}{ Variable } & \multicolumn{4}{|c|}{ Appropriate } & \multirow{3}{*}{ P-value } \\
\hline & \multicolumn{2}{|c|}{ Yes } & \multicolumn{2}{|c|}{ No } & \\
\hline & No. & $\%$ & No. & $\%$ & \\
\hline \multicolumn{6}{|c|}{ System } \\
\hline Upper respiratory tract & 11 & 3.1 & 347 & 96.9 & \multirow{10}{*}{$<0.001$} \\
\hline Urinary tract & 110 & 37.9 & 180 & 62.1 & \\
\hline Skin \& soft tissue & 63 & 25.3 & 186 & 74.7 & \\
\hline Gastrointestinal tract & 24 & 17.5 & 113 & 82.5 & \\
\hline No mentioned diagnosis & 0 & 00.0 & 118 & 100.0 & \\
\hline Genital tract & 4 & 7.8 & 47 & 92.2 & \\
\hline Lower respiratory tract & 13 & 43.3 & 17 & 56.7 & \\
\hline Maxillofacial and oral & 5 & 18.5 & 22 & 81.5 & \\
\hline Musculoskeletal & 6 & 23.1 & 20 & 76.9 & \\
\hline Others $^{a}$ & 1 & 3.7 & 26 & 96.3 & \\
\hline \multicolumn{6}{|c|}{ Prescribing physician's specialty } \\
\hline Emergency & 150 & 14.5 & 881 & 85.5 & \multirow{5}{*}{$<0.001$} \\
\hline Obstetrics and Gynecology & 62 & 31.6 & 134 & 68.4 & \\
\hline General Surgery & 11 & 25.6 & 32 & 74.4 & \\
\hline Internal Medicine & 7 & 46.7 & 8 & 53.3 & \\
\hline Other specialties ${ }^{b}$ & 7 & 25.0 & 21 & 75.0 & \\
\hline
\end{tabular}

a Including: Ophthalmology, central nervous system, hematology, oncology and infectious disease.

b Including: Ear, nose and throat, orthopedic, plastic, maxillofacial and oral, urology, neurology.

clavulanic acid ( $n=193 ; 14.7 \%)$, followed by others antibiotics are shown in Table 3. A total of 112 patients received single intravenous antibiotic dose only and without additional oral antibiotic course. Another 46 patients received ceftriaxone, followed by cefuroxime $(n=39)$, metronidazole $(n=26)$ and meropenem $(n=1)$.

Chi squared $p$ value showed a high significant difference in appropriateness percentage between different presenting infections and prescribed medical specialties. Inappropriateness was more in those without mentioned diagnosis ( $n=118$; 100\%) followed by upper respiratory tract infection ( $n=347 ; 96.9 \%)$. Antibiotic therapy was found to be inappropriate in 1076 prescriptions (81.9\%) of the total prescriptions (1313). The most common cause for inappropriateness was the unjustified use
( $n=476 ; 36.3 \%$ ) as demonstrated in Table 4. Chisquared $p$ value showed that there is a high statistical significant difference in appropriateness percentage between males and females; antibiotics were used less inappropriately among females ( $n=475 ; 76.2 \%$ ) than male ( $n=601 ; 87.1 \%$ ) (Table 4). Appropriate and inappropriateness of chemotherapy to each used antimicrobial drug is shown in Table $\mathbf{5}$.

\section{Discussion}

The major finding of this study was the presence of high levels of inappropriate use of antibiotics. Although the principals of antibiotic prescriptions have been well established internationally for many years, their inappropriate use is still common 
Table 3. Frequency and percentages of various antibiotics prescribed in the emergency department ${ }^{\mathrm{a}}$

\begin{tabular}{|c|c|c|}
\hline Antimicrobial prescribed & No. & $\%$ \\
\hline Cefuroxime & 492 & 37.5 \\
\hline Ciprofloxacin & 273 & 20.8 \\
\hline Cephalexin & 126 & 9.6 \\
\hline Amoxicillin/clavulanate acid & 193 & 14.7 \\
\hline Metronidazole & 101 & 7.7 \\
\hline Amoxicillin & 57 & 4.3 \\
\hline Clarithromycin & 18 & 1.4 \\
\hline Erythromycin & 9 & 0.7 \\
\hline Cloxacillin & 12 & 0.9 \\
\hline Doxycycline & 9 & 0.7 \\
\hline Nitrofuranton & 2 & 0.2 \\
\hline Norfloxacin & 1 & 0.1 \\
\hline Trimethoprim-sulfamethaxazole & 1 & 0.1 \\
\hline Trimethoprim-sulfamethaxazole DS & 1 & 0.1 \\
\hline
\end{tabular}

a Some of the patients used more than one antibiotic. especially in the developing countries[15,16]. The study also revealed that the antibiotic prescription rate in the emergency room is $7.2 \%$, which is low compared to other countries in our region, while such rate was $61.9 \%$ in Iran [17], 60.7\% in England [18], 48\% in Norway [19] and $46 \%$ in Saudi Arabia [20].

It has been reported that penicillin and flouroquinolones were the most frequently prescribed antibiotics in several studies in the developed and developing countries [16,21-22].

In this study cephalosporins, specifically cefuroxime was the most prescribed antibiotic, because of their broad spectrum of activity, clinical efficacy, availability and favorable tolerability profiles. However, penicillin was much less prescribed compared with other countries despite their availability. The flouroquinolones were also less prescribed than in other studies because use of these antibiotics are restricted by the antibiotic policy of hospital. Similar findings were found in a

Table 4. Appropriateness in relation to demographical data $(n=1313)$

\begin{tabular}{|c|c|c|c|c|c|}
\hline \multirow{2}{*}{ Characteristics } & \multicolumn{2}{|c|}{ Appropriate } & \multicolumn{2}{|c|}{ Inappropriate } & \multirow{2}{*}{ P-value } \\
\hline & No. & $\%$ & No. & $\%$ & \\
\hline \multicolumn{6}{|c|}{ Gender } \\
\hline Male & 89 & 12.9 & 601 & 87.1 & \multirow{2}{*}{$<0.001$} \\
\hline Female & 148 & 23.8 & 475 & 76.2 & \\
\hline \multicolumn{6}{|c|}{ Age group in years } \\
\hline $14-30$ & 108 & 18.2 & 487 & 81.8 & \multirow{5}{*}{0.806} \\
\hline $31-40$ & 57 & 16.8 & 283 & 83.2 & \\
\hline $41-50$ & 27 & 17.5 & 127 & 82.5 & \\
\hline $51-60$ & 19 & 17.4 & 90 & 82.6 & \\
\hline$>60$ & 26 & 22.6 & 89 & 77.4 & \\
\hline \multicolumn{6}{|c|}{ Nationality } \\
\hline Bahraini & 220 & 18.0 & 1004 & 82.0 & \multirow{2}{*}{0.997} \\
\hline Non Bahrainis & 17 & 19.1 & 72 & 80.9 & \\
\hline
\end{tabular}


Table 5. Appropriate and inappropriateness of antibiotic therapy.

\begin{tabular}{|c|c|c|c|c|c|c|c|c|c|c|c|}
\hline \multirow{3}{*}{ Antimicrobial } & \multirow{2}{*}{\multicolumn{2}{|c|}{ appropriate }} & \multicolumn{8}{|c|}{ inappropriate } & \multirow{3}{*}{ Total } \\
\hline & & & \multicolumn{2}{|c|}{ total } & \multicolumn{2}{|c|}{$\begin{array}{l}\text { inappropriate } \\
\text { choice }\end{array}$} & \multicolumn{2}{|c|}{ Not indicated } & \multicolumn{2}{|c|}{$\begin{array}{c}\text { Wrong } \\
\text { combination }\end{array}$} & \\
\hline & $\mathrm{n}$ & $\%$ & n & $\%$ & $\mathbf{n}$ & $\%$ & $n$ & $\%$ & n & $\%$ & \\
\hline Cephalexin & 80 & 63.49 & 46 & 36.51 & 14 & 11.11 & 31 & 24.60 & 1 & 0.79 & 126 \\
\hline Ciprofloxacin & 134 & 49.08 & 139 & 50.92 & 25 & 9.16 & 101 & 37.00 & 13 & 4.76 & 273 \\
\hline Metronidazole & 16 & 15.84 & 85 & 84.16 & 15 & 14.85 & 59 & 58.42 & 11 & 10.89 & 101 \\
\hline clarithromycin & 11 & 61.11 & 7 & 38.89 & 1 & 5.56 & 2 & 11.11 & 4 & 22.22 & 18 \\
\hline Erythromycin & 1 & 11.11 & 8 & 88.89 & 2 & 22.22 & 2 & 22.22 & 4 & 44.44 & 9 \\
\hline Amoxicillin & 36 & 63.16 & 21 & 36.84 & 0 & 0.00 & 21 & 36.84 & 0 & 0.00 & 57 \\
\hline Cefuroxime & 273 & 55.49 & 219 & 44.51 & 53 & 10.77 & 156 & 31.71 & 10 & 2.03 & 492 \\
\hline $\begin{array}{l}\text { Amoxicillin/ } \\
\text { clavulanate }\end{array}$ & 125 & 64.77 & 68 & 35.23 & 15 & 7.77 & 51 & 26.42 & 2 & 1.04 & 193 \\
\hline Cloxacillin & 7 & 58.33 & 5 & 41.67 & 2 & 16.67 & 3 & 25.00 & 0 & 0.00 & 12 \\
\hline Doxycycline & 3 & 33.33 & 6 & 66.67 & 1 & 11.11 & 2 & 22.22 & 3 & 33.33 & 9 \\
\hline Nitrofuranton & 0 & 0.00 & 2 & 100.00 & 1 & 50.00 & 1 & 50.00 & 0 & 0.00 & 2 \\
\hline Norfloxacin & 0 & 0.00 & 1 & 100.00 & 0 & 0.00 & 1 & 100.00 & 0 & 0.00 & 1 \\
\hline $\begin{array}{c}\text { Trimethoprim/ } \\
\text { sulphamethaxazole }\end{array}$ & 0 & 0.00 & 1 & 100.00 & 0 & 0.00 & 1 & 100.00 & 0 & 0.00 & 1 \\
\hline $\begin{array}{c}\text { Trimethoprim/ } \\
\text { sulphamethaxazole } \\
\text { DS }\end{array}$ & 1 & 100.00 & 0 & 0.00 & 0 & 0.00 & 0 & 0.00 & 0 & 0.00 & 1 \\
\hline
\end{tabular}

study done in Bangladesh, where cephalosporines were used in $31.78 \%$ of all prescriptions and followed the use of macrolides (27.33\%) [23]. A study in France showed cephalosporines were used in $21 \%$ and flouroquinolones in $22 \%$ in treatment of the patients, respectively, while penicillins used in $43 \%$ [24]. Amoxicillin-clavulanate was used in 30\% of the patients in Saudi Arabia [20], while its use reached $54 \%$ in Spain [25]. A study performed in the United States reported that azithromycin was the mostly prescribed antibiotic for outpatients[26].

This study indicated that emergency doctors in our hospital have misused intravenous administration of antibiotics in patients without clearly indication for their need. Inappropriate intravenous therapy increases the cost of care and also exposes the patients to the risk of infection associated with intravenous catheters [27].

Overall our study found that antibiotic therapy was inappropriate in $80.9 \%$ of the examined patients, despite the accessibility of the antibiotic guidelines in the emergency rooms in form of booklets and on the internet. However, our results show antibiotics inappropriateness rate was much lower than in studies done in other countries, including Australia [28], Spain [25], France [29] 48.6\%, 43\%, 31\%, respectively.

The high rate of the inappropriate use of antibiotics 
in our study was mostly due to unjustified use and the wrong duration of treatment. In Spain similar results were obtained from a study found that $40 \%$ of patients were prescribed antibiotics without justified cause [25]. Another study in Spain showed most cause of inappropriateness is due to improper duration of treatment (39.9\%) [21]. A study in the Saudi Arabia showed that the duration of treatment was the most common cause of inappropriateness [30].

The present study shows that the burden of respiratory symptoms / conditions in our emergency department was large, and there was marked misuse in antimicrobial prescriptions.

Our quality evaluation showed that the rate of inappropriate prescription of antibiotics were higher among the emergency room doctors than other medical specialties. Antimicrobial therapy should be tailored to each patient taking into consideration the presenting signs and symptoms, the local patterns of antimicrobial resistance and the potential for collateral damage associated with antimicrobial use. Selecting the correct drug, dose, and shortest clinically effective duration of therapy are important keys to optimal antimicrobial stewardship. Most of the obstacles could be addressed and controlled by implementing a well-structured program for the emergency department in order to minimize the inappropriate prescription of antibiotics and, subsequently to control the rate of developing antimicrobial resistant organisms.

There are some limitations of this study: First, the level of the antibiotic use could not be accurately measured due to the absence of accurate medication charts and the poor quality of medication recordkeeping in the emergency department. Second, our study was based on the evaluation of the emergency record of the patients and not based on the clinical evaluation of the patients. It is the responsibility of all healthcare providers to practice antimicrobial stewardship and prescribe antimicrobials wisely and rationally in order to minimize the inappropriate prescription of antibiotics and subsequently the rate of the resistant organisms. It is time to incorporate emergency department in the antibiotic stewardship program in our hospital policies. There is also a great full structured educational program available for the emergency room physicians and other specialty doctors to ensure better compliance with the local and international guidelines.

In conclusion, our results revealed a significantly high level of the inappropriate use of antibiotics in the emergency department in Salmaniya Medical Complex, being highest among emergency doctors. Cefuroxime was the most prescribed antibiotic followed by ciprofloxacin. Most common cause of inappropriateness is the unjustified use and improper duration of antibiotic treatment.

\section{References}

1. Gholamreza-Sepehri, and Meimandi MS. The quality of prescribing in general practice in Kerman, Iran. Int J Health Care Qual Assur Inc Leadersh Health Serv. 2005;18:353-60.

2. World Health Organization. The evolving threat of antimicrobial resistance. Executive summary. World Health Organization, Geneva; 2012. Available at: http://apps.who.int/iris/bitstream/ 10665/75389/1/WHO_IER_PSP_2012.2_eng.pdf

3. Chief Medical Officer. Annual Report of the Chief Medical Officer. Volume 2, 2011. Infections and the rise of antimicrobial resistance. London: Department of Health, 2013.

4. May L, Cosgrove S, L'Archeveque M, Talan DA, Payne P, Rothman RE. Antimicrobial Stewardship in the Emergency Department and Guidelines for Development. Annals of emergency medicine 2013;62(1):10.1016 j.annemergmed.2012.09.002.

5. Shehab N, Patel PR, Srinivasan A, Budnitz DS. Emergency department visits for antibiotic-associated adverse events. Clin Infect Dis 2008; 15;47(6):735-43.

6. Budnitz DS, Pollock DA, Mendelsohn AB, Weidenbach KN, McDonald AK, Annest JL. Emergency department visits for outpatient adverse drug events: demonstration for a national surveillance system. Ann Emerg Med 2005; 45(2):197-206.

7. Patel P., Zed P.J. Drug-related visits to the emergency department: how big is the problem? Pharmacotherapy 2002; 22(7):915-923.

8. Al-Niemat SI, Aljbouri TM, Goussous LS, Efaishat RA, Salah RK. Antibiotic Prescribing Patterns in Outpatient Emergency Clinics at Queen Rania Al Abdullah II Children's Hospital, Jordan, 2013. Oman Med J 2014;29(4):250-254

9. Cotta MO, Robertson MS, Upjohn LM, Marshall C, Liew D, Buising KL. Using periodic point-prevalence surveys to assess 
appropriateness of antimicrobial prescribing in Australian private hospitals. Intern Med J 2014;44:240-6.

10. Duran C, Marques S, Hernandez S, Trenchas V, García JJ, Luaces C. Antibiotic prescribing in a paediatric emergency department. Ann Pediatr (Barc). $2010 ; 73(3): 115-$

11. Schafermeyer RW, Asplin BR. Hospital and emergency department crowding in the United States. Emerg Med (Fremantle) 2003;15(1):22-27.

12. Jameela Al Salman. Antimicrobial Guidelines.2013, 20-37. Ministry of Health, Bahrain.

13. Infectious Diseases Society of America. IDSA practice guidelines. Available at: http://www.idsociety.org/idsa_practice_guidelines (accessed on July 6, 2015)

14. DePestel DD, Eiland EH, Lusardi K, Destache CJ, Mercier RC,et al. Clin Infect Dis. 2014;15;59 Suppl 3:S154-61.

15. Blomberg B. Antimicrobial resistance in developing countries. Tidsskr Nor Laegeforen. 2008;128:2462-2466.

16. Byarugaba DK. A view on antimicrobial resistance in developing countries and responsible risk factors. Int J Antimicrob Agents, 2004; 24: 105-110.

17. Moghadamnia A, Mirbolooki MR, Aghili MB. General practitioner prescribing patterns in Babol city, Islamic Republic of Iran. East Mediterr Health J 2002;8(4-5):550-55

18. Majeed A, Moser K. Age- and sex-specific antibiotic prescribing patterns in general practice in England and Wales in 1996. Brit J Gen Pract 1999;49(446):735-736.

19. Lindbaek M, Berild D, Straand J, and Hjortdahl P. Influence of prescription patterns in general practice on anti-microbial resistance in Norway. Brit J Gen Pract 1999;49(443):436-440.

20. Oqal MK, Elmorsy SA, Alfhmy AK, Alhadhrami RM, Ekram RA, Althobaiti IA, et al. Patterns of antibiotic prescriptions in the outpatient department and emergency room at a Tertiary Care Center in Saudi Arabia. Saudi J Med Med Sci 2015;3:124-9

21. Laguna P, Moya MS, García F, Salgado R, and Calabrese S. Utilization of antibiotics in a hospital emergency department. Quality of prescription. Rev Clin Esp. 1996 ;196(7):431-6.

22. Al-Niemat SI, Bloukh DT, Al-Harasis MD, Al-Fanek AF, Salah RK. Drug use evaluation of antibiotics prescribed in a Jordanian hospital outpatient and emergency clinics using WHO prescribing indicators. Saudi Med J 2008;29(5):743-8.

23. Biswas M, Roy DN, Tajmim A, et al. Prescription antibiotics for outpatients in Bangladesh: a cross-sectional health survey conducted in three cities. Ann Clin Microbiol and Antimicrob. 2014; 13:15

24. Asseray $N$, Bleher $Y$, Poirier $Y$, Hoff J, Boutoille D, Bretonniere $C$, et al. Use of antibiotics in emergency units: qualitative and quantitative assessment. Med Mal Infect. 2009 ;39(3):203-8.

25. Ramos Martínez A, Cornide Santos I, Marcos García R, and Calvo Corbella E. Antibiotic prescription quality at a hospital emergency service. An Med Interna 2005 ;22(6):266-70.

26. Lauri AH, Monina GB, Rebecca MR, Katie J. Suda, Robert J, et al. US Outpatient

27. Antibiotic Prescribing Variation According to Geography, Patient Population, and Provider Specialty in 2011. Clin Infect Dis. (2015) doi: 10.1093/cid/civ076.
28. Finch RG, Metlay JP, Davey PG, Baker LJ. Educational interventions to improve antibiotic use in the community: report from the International Forum on Antibiotic Resistance (IFAR) colloquium, 2002. Lancet Infect Dis 2004; 4: 44-53.

29. Cotta MO, Robertson MS, Upjohn LM, Marshall C, Liew D, Buising $\mathrm{KL}$. Using periodic point-prevalence surveys to assess appropriateness of antimicrobial prescribing in Australian private hospitals. Intern Med J 2014 Mar;44(3):240-6.

30. Gennai S, Pavese P, Vittoz JP, Decouchon C, Remy S, Dumont O, et al. Assessment of antibiotic prescriptions in the emergency department of a general hospital. Prospective analysis of 211 prescriptions. Presse Med. 2008;37(1 Pt 1):6-13. Epub 2007 Nov 5.

31. Alanazi MQ, Al-Jeraisy MI, and Salam M. Prevalence and predictors of antibiotic prescription errors in an emergency department, Central Saudi Arabia. Drug Healthc Patient Saf. 2015;4;7:103-11

\section{Comment on this article:}

\section{(f) $B$ in $8+\mathbf{S} P$}

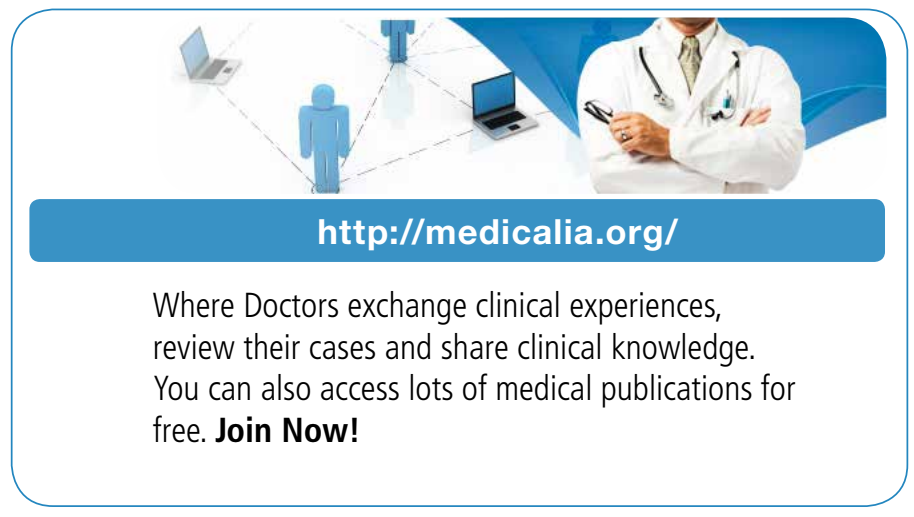

\section{Publish with iMedPub}

http://www.imed.pub

The Journal is an open access peer-reviewed journal that publishes scientific papers about all aspects of antimicrobials. The journal will publish original research articles, reviews, brief reports and case reports dealing with basic and clinical antibacterial agents, antiviral, antiprotozoals, antituberculuous, antifungal and antihelminthes agents.

All manuscripts must be prepared in English, and are subject to a rigorous and fair peer-review process. Accepted papers will immediately appear online.

The journal aims to advance the knowledge, attitude and the research of chemotherapy in the Arabic world in cooperation with international, national scientific and public societies as well as research centers with similar aims and objectives. 